\title{
Emergent mechanical thrombectomy for right atrial clot and massive pulmonary embolism using FlowTriever.
}

\author{
Jason Salsamendi ${ }^{1}$, Pilar Bayona Molano ${ }^{2}$, and Naganathan Mani ${ }^{3}$ \\ ${ }^{1}$ Kaiser Permanente \\ ${ }^{2}$ Washington University in Saint Louis \\ ${ }^{3}$ Mallinckrodt Institute of Radiology
}

September 16, 2020

\begin{abstract}
Right heart thrombus and pulmonary embolism is a condition that requires emergent treatment with reported fatal outcomes. Endovascular mechanical thrombectomy of right heart clot has been described with the use of AngioVac. We describe the use of FlowTriever device as a feasible alternative to treat clot in transit.
\end{abstract}

\section{TEXT}

\section{Introduction}

Alternative therapies to massive or submassive pulmonary embolism (PE) include surgical embolectomy, systemic thrombolysis, and endovascular catheter directed lysis. In patients with contraindication to thrombolysis, mechanical thrombectomy can restore pulmonary circulation and avoid cardiogenic shock. Surgical thrombectomy is a tool for patients with rapid hemodynamic deterioration, clot-in-transit within the right heart, or when an underlying right-to-left shunt is present (1). The 2011 American Heart Association guidelines for surgical embolectomy included patients with massive PE who exhibited contraindications to receive fibrinolysis, or patients who remain unstable after thrombolysis (2). Reported complications of the surgical approach included rethrombosis risk, postoperative bleeding, cardiac tamponade, and sternal wound infection (3).

The AngioVac device (Angiodynamics, Latham, NY USA), offers an important alternative to surgical thrombectomy for right heart thrombus (RiHT) with a success rate reported up to $87 \%$ in a literature review including 28 patients (4). However, the reported success for pulmonary embolism (PE) was significantly lower at $12.5 \%$. The complication rate was up to $12 \%$ (Total of 56 patients treated) including fatal retroperitoneal hemorrhage or right ventricular perforation $(4,5)$. A recent case report described the use of FlowTriever under transthoracic echocardiography (TTE) guidance for mechanical thrombectomy of right intra-atrial thrombus with instantaneous and marked decrease in clot burden and pulmonary reperfusion and hemodynamic recovery. It also described the benefit of less blood loss than the AngioJet and Penumbra systems (6). We report the use of FlowTriever device as a modality to treat clot in transit and massive PE when thrombolysis is contraindicated.

\section{Case Report}

A 53 year old male with a past medical history of high-grade glioma and recent brain surgery presented to ED after a syncopal episode. His head computed tomography (CT) was negative for intracranial hemorrhage or infarct. Simultaneous Chest CT- PE protocol (2 mm slices) noted large saddle pulmonary embolism with significant clot burden (Figure 1a, b). It revealed a "snake like" thrombus in the right atrium suggesting free 
floating clot with significant right heart $(\mathrm{RH})$ strain (Figure 2a, b). The N-terminal pro-B-type natriuretic peptide (NT-proBNP) was $832 \mathrm{pg} / \mathrm{mL}$ (Ref Range: $<=300 \mathrm{pg} / \mathrm{mL}$ ), and Troponin I $0.91 \mathrm{ng} / \mathrm{mL}$ (Ref Range: $0.00-0.03 \mathrm{ng} / \mathrm{mL}$ ). Bilateral lower extremities venous Doppler's reported acute vein thrombosis in the left popliteal vein. The patient was transferred to the intensive care unit (ICU) with a heart rate (HR) of 127 beats/min, systolic blood pressure of $120 \mathrm{mmHg}$, and peripheral capillary oxygen saturation $\left(\mathrm{SpO}_{2}\right)$ of $92 \%$ on 6 liters of oxygen in a non-rebreather. Full anticoagulation with unfractionated Heparin was started. The use of thrombolysis was precluded given his neurological status. Interventional radiology and cardiothoracic surgery (CTS) were consulted for possible mechanical thrombectomy of pulmonary embolism and clot in the right atrium (RA).

The patient was sedated and monitored by cardiovascular anesthesia. Additional venous and arterial 6 Fr vascular sheaths were inserted to anticipate any urgent transition to extracorporeal membrane oxygenation (ECMO). Through a right common femoral vein approach, a catheter was advanced into the inferior vena cava (IVC). IVCgram demonstrated clot extending from the IVC into the RA (Figure 3a, b). Given the potential risk of dislodging clot that could increase the PA pressure, the Triever20 catheter (Inari Medical Inc., Irvine, CA, USA) was inserted for mechanical thrombectomy. Suction thrombectomy was performed at the level of the IVC confluence and RA under fluoroscopic guidance. Moderate amount of clot was aspirated (Figure 4) and resolution was confirmed with a follow up cavogram. The main PA was accessed using a $6 \mathrm{Fr}, 100 \mathrm{~cm}$ Vert catheter. The initial main PA pressure was elevated $(46 / 13 \mathrm{mmHg}$, mean of $38 \mathrm{mmHg}$ ). Pulmonary angiography demonstrated extensive saddle embolism (Figure 5a, b, c). Next, the Triever20 catheter was advanced to the left PA and a large amount of clot was retrieved from the segmental branches with minimal residual thrombus. The self-expanding nitinol disks of the device were not required given the results obtained with aspiration. The same intervention was repeated in the main and right PA with improvement of PA pressures $(28 / 9 \mathrm{mmHg}$, mean $14 \mathrm{mmHg})$ and oxygen demand. The blood loss was approximately $150 \mathrm{cc}$ and there was no change in the hemoglobin monitored throughout and after the procedure.

Follow-up chest CT-PE protocol demonstrated decreased clot burden in the RA and PA without visualization of right heart strain (Figure 6a, b). Subsequent TTE reported no definite RA thrombus. Walking oxygen assessment showed resting Sp02 of 96\% and walking Sp02 100\% on room air. Four days later after the procedure, the patient was discharged on long term Apixaban.

\section{Discussion}

RiHT are classified as type A Thrombi, those serpiginous, highly mobile, and with high mortality. Type B Thrombi are less mobile, pedunculated, broad base, and have better prognosis. And type $\mathrm{C}$ is an intermediate in all characteristics. (7). The association of free floating-right heart thrombi and massive pulmonary embolism (PE) has an incidence of $4-18 \%$ (1). It is a life-threatening condition that requires emergent diagnosis and treatment with reported fatal outcomes up to $21 \%$ in 14 days (8). Thrombi that are adherent to the RA or right ventricle (RV) wall usually have a more benign prognosis (8). However, free-floating, "snake-like" thrombi are uncommon and usually associated with massive unstable pulmonary embolism (9, 10). Some studies have reported the clot in transit as a predictor of poor outcome (11). There are no evidence-based guidelines for treatment of pulmonary embolism complicated by free-floating RH thrombus (12).

In a retrospective analysis of 177 cases of right heart thromboembolism and pulmonary embolism in $98 \%$ of the cases, the mortality rate associated with no therapy, anticoagulation therapy, surgical embolectomy, and thrombolysis was $100.0 \%, 28.6 \%, 23.8 \%$, and $11.3 \%$, respectively (13). One year later the European Cooperative Study reported a mortality rate of $60 \%$ for anticoagulated patients; $40 \%$ for those treated with thrombolytics; and $27 \%$ for those submitted to surgical procedures, which suggested the surgical approach to be the most effective (8). Although, some small case series of RiHT type A showed thrombolytic therapy with a favorable outcome in relation to mortality (7), some controversy has arisen with systemic thrombolysis in the treatment of giant right atrial thrombosis and clot fragmentation, with subsequent fatal results related to the development of cardiogenic shock (14). Until 2017, there was no studies that clearly determined which 
patients with $\mathrm{PE}$ and RiHT would benefit from reperfusion treatment (15). With this purpose, the Registro Informatizado de la Enfermedad Trombo Embolica (RIETE) Registry compared the outcome during the first month after treatment of standard anticoagulation therapy (heparin) against reperfusion treatment (including thrombolysis or cardiac surgery). The study did not show differences in survival among patients who received standard anticoagulation compared with the reperfusion therapy (15). They concluded that reperfusion therapies might be reserved for patients who have acute symptomatic pulmonary embolism, associated hypotension, or shock irrespective of the presence or absence of coexisting RiHT (15).

Few reports of percutaneous treatment of free-floating thrombus in the right atrium and $\mathrm{PE}$ has been published, and their results have been promising. In these cases, several types of catheters were used in which they also included direct AngioVac and IVC filters, or a Cook intravascular retrieval. $(16,17,18,19)$.

FlowTriever System, an FDA approved device for treatment of PE, allows it to be maneuvered into the pulmonary segmental branches and to aspirate central and more distal pulmonary clot. It permits the use of contrast injections through a coaxial system which enables us to monitor the progress of the intervention. In the multicenter single-arm FLARE (FlowTriever Pulmonary Embolectomy Clinical Study), presented in 2018 on 106 patients with intermediate-risk PE who underwent catheter embolectomy using the FlowTriever catheter, they found the system safe and effective. There was a significant improvement in RV/LV ratio and minimal major bleeding. Potential advantages included immediate thrombus removal, absence of thrombolytic complications, and reduced need for post-procedural critical care (20).

This report highlights the effectiveness of FlowTriver for mechanical thrombectomy of RiHT and PE in this case. Limitations described for AngioJet or AngioVac as dysrhythmias, or difficult maneuverability were not found (6). Additionally, its use showed a rapid normalization of PA pressure and Sp02. This method allowed removal of the clot in a high risk patient who exhibited contraindications to thrombolysis. Indigo CAT (Penumbra) device has been evolving to larger diameters which can be manipulated at a more segmental level and it is a very useful alternative for pulmonary embolism however, the use on Right, has not been reported.

\section{Conclusion}

It is feasible to perform mechanical thrombectomy using Flowtriever device in the presence of PE with concomitant clot in transit. FlowTriever permits the simultaneous injection of contrast to evaluate the residual clot burden under fluoroscopic guidance. It requires further investigation to define it as a frontline tool.

\section{References}

1. Athappan G, Sengodan P, Chacko P, Gandhi S. Comparative efficacy of different modalities for treatment of right heart thrombi in transit: a pooled analysis. Vasc Med 2015;20:131-8.

2. Jaff M R, McMurtry M S, Archer S L et al. Management of massive and submassive pulmonary embolism, iliofemoral deep vein thrombosis and chronic thromboembolic pulmonary hypertension: a scientific statement from the American Heart Association. Circulation. 2011;123(16):1788-1830.

3. Moriarty JM, Edwards M, Plotnik AN. Intervention in Massive Pulmonary Embolus: Catheter Thrombectomy/Thromboaspiration versus Systemic Lysis versus Surgical Thrombectomy. Seminars in Interventional Radiology. 2018;35(2):108-115. DOI: 10.1055/s-0038-1642039.

4. Worku B, Salemi A, D’Ayala MD, Trambaugh R, Girardi LN, Gulkarov I M. The AngioVac Device Understanding the Failures on the Road to Success. Innovations (Phila). 016;11(6):430- 433. doi:10.1097/IMI.0000000000000310

5. Al-Hakim R, Park J, Bansal A, Genshaft S, Moriarty J M. Early experience with AngioVac aspiration in the pulmonary arteries. J Vasc Interv Radiol. 2016;27(05):730-734

6. Nezami N, Latich I, Murali N, Rahmat A, Lin BA, Mohammadreza S, Pollak J, Hamid M. Right Atrial and Massive Pulmonary Artery Mechanical Thrombectomy under Echocardiography Guidance Using the FlowTriever System. EJVES Short Reports 2019; 45, 22e25. 
7. Kajaohideen N, Swaminathan N, Palanisamy G, et al. Morphology of right heart thrombi in Pulmonary Embolism: A case series and analysis. J Indian Acad Echocardiogr Cardiovasc Imaging 2017; 1:24-26.

8. Torbicki A, Galié N, Covezzoli A, et al. Right heart thrombi in pulmonary embolism: results from the International Cooperative Pulmonary Embolism Registry. J Am Coll Cardiol 2003; 41(12): 2245-2251.

9. Kronik G. The European cooperative study on the clinical significance of right heart thrombi. Eur Heart J 1989;10:1046-1059.

10. Naeem K. Floating thrombus in the right heart associated with pulmonary embolism: The role of echocardiography. Pak J Med Sci 2015;31(1):233-235. doi: http://dx.doi.org/10.12669/pjms.311.6219.

11. Kostrubiec M, Elikowski, et al. Outcome of patients with right heart thrombi: the Right Heart Thrombi European Registry. Eur Respir J 2016; 47 (2016), pp. 869-878

12. Dalen JE. Free-floating right heart thrombi. Am J Med 2017; 130(5): 501.

13. Rose S, Punjabi NM, Pearse DB. Treatment of Right Heart Thromboemboli. Chest 2002; 121 (3): 806-814. DOI:https://doi.org/10.1378/chest.121.3.806

14. Ruiz-Bailén M, López-Caler C, Castillo-Rivera A, et al. Giant right atrial thrombi treated with thrombolysis. Can J Cardiol 2008;24(4):312-314. doi:10.1016/s0828-282x(08)70183-1.

15. Barrios D, Chavant J, Jiminez D, et al. Treatment of right heart thrombi associated with acute pulmonary embolism. Am J Med 2017; 130: 588-595

16. Momose, T, Morita T, Misawa T. Percutaneous treatment of a free-floating thrombus in the right atrium of a patient with pulmonary embolism and acute myocarditis. Cardiovasc Interv and Ther 2013;28(2), 188-192. https://doi.org/10.1007/s12928-012-0141-0

17. Otoupalova E, Dalal B, Renard B. Right heart thrombus in transit: a series of two cases. Crit Ultrasound J 2017;9(1):14. doi:10.1186/s13089-017-0069-9.

18. Davies RP, Harding J, Hassam R. Percutaneous retrieval of a right atrioventricular embolus. Cardiovasc Intervent Radiol 1998; 21: 433-435. https://doi.org/10.1007/s002709900295.

19. Nickel B, McClure T, Moriarty J. A Novel Technique for Endovascular Removal of Large Volume Right Atrial Tumor Thrombus. Cardiovasc Intervent Radiol 2015;38(4):1021-1024. doi:10.1007/s00270-0140986-y.

20. Tu T, Toma C, Tapson VF, et al. A Prospective, Single-Arm, Multicenter Trial of Catheter-Directed Mechanical Thrombectomy for Intermediate-Risk Acute Pulmonary Embolism. The FLARE Study. JACC Cardiovas Interv 2019; 12(9):859-869. DOI: 10.1016/j.jcin.2018.12.022

\section{Hosted file}

TTMaindocument-FlowTriever--1--Corrected.docx available at https://authorea.com/users/346101/ articles/481419-emergent-mechanical-thrombectomy-for-right-atrial-clot-and-massivepulmonary-embolism-using-flowtriever

\section{Hosted file}

Title-and-Authors.docx available at https://authorea.com/users/346101/articles/481419emergent-mechanical-thrombectomy-for-right-atrial-clot-and-massive-pulmonary-embolismusing-flowtriever

\section{Hosted file}

TTMaindocument_FlowTriever (1) Corrected.docx available at https://authorea.com/users/346101/ articles/481419-emergent-mechanical-thrombectomy-for-right-atrial-clot-and-massivepulmonary-embolism-using-flowtriever

\section{Hosted file}

Figures.docx available at https://authorea.com/users/346101/articles/481419-emergentmechanical-thrombectomy-for-right-atrial-clot-and-massive-pulmonary-embolism-usingflowtriever 